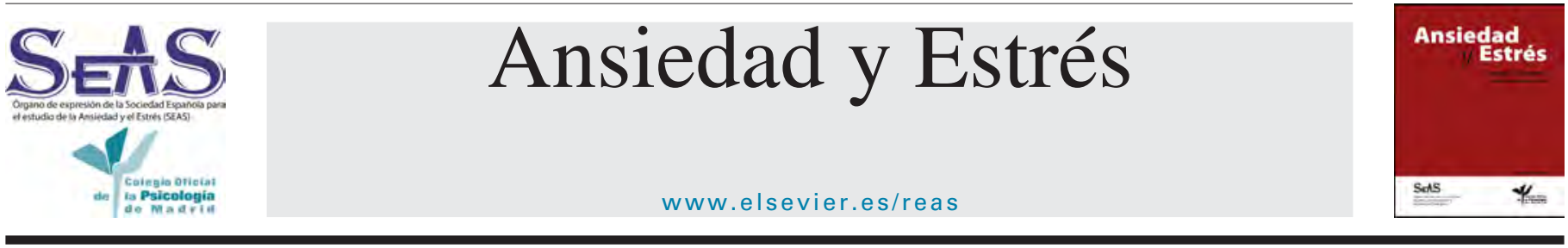

Original

\title{
Propiedades psicométricas del Inventario de Situaciones y Respuestas de Ansiedad Breve (ISRA-B)
}

\author{
Antonio Cano-Vindel ${ }^{\mathrm{a}}$, Roger Muñoz-Navarro ${ }^{\mathrm{b}, *}{ }$, Luciana S. Moretti $^{\mathrm{c}}$ y Leonardo A. Medrano ${ }^{\mathrm{c}}$ \\ a Universidad Complutense de Madrid, España \\ b Universidad de Zaragoza, España

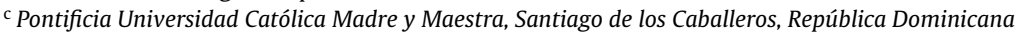

\section{INFORMACIÓN DEL ARTÍCULO}

\section{Historia del artículo:}

Recibido el 24 de julio de 2020

Aceptado el 31 de julio de 2020

On-line el xxx

\section{Palabras clave:}

Ansiedad

Evaluación

Estructura factorial

ISRA

\begin{abstract}
R E S U M E N
Introducción y objetivos: La ansiedad es la reacción emocional más estudiada, que nos pone en alerta ante la posibilidad de obtener un resultado negativo, por lo que resulta adaptativa en la gran mayoría de las ocasiones, aunque también está en la base de los trastornos emocionales más prevalentes. Por ello, su evaluación ha generado mucha investigación y transferencia. El Inventario de Situaciones y Respuestas de Ansiedad (ISRA) fue desarrollado en España, incluye 22 situaciones (S) y 24 respuestas (R) de ansiedad, que al cruzarlas se forman 224 reactivos (del tipo «en tal S, experimento esta R»). Desde hace más de tres décadas ha sido ampliamente utilizado en la investigación, así como en la práctica, y se han publicado estudios sobre sus propiedades psicométricas en diferentes países. Una versión más breve (ISRA-B) de 46 ítems, sin contenidos cruzados $\mathrm{S} \times \mathrm{R}$, sino una escala de $22 \mathrm{~S}$ y otra de $24 \mathrm{R}$, con las que evalúan las mismas ocho facetas de la ansiedad, ha mostrado buenas propiedades psicométricas también; sin embargo, existen menos publicaciones psicométricas en población española. El objetivo de este estudio fue analizar las propiedades psicométricas del ISRA-B en España.

Método: Para ello se contó con 3,346 voluntarios (36.4\% hombres), con edades de 15 a 94 años (media: 30.3; DE: 14.6).

Resultados: Los resultados confirmaron excelente consistencia interna para Total (.94) y subescala $\mathrm{R}(.91)$, buena para S (.89) y sistemas de respuesta Fisiológico (.86) y Cognitivo (.84), y aceptable para Motor (.70). También se encontró buena estabilidad test-retest al cabo de un mes (.78) y buena validez externa al correlacionar 87 con el ISRA original. La estructura factorial del ISRA-B obtenida se adecuó al modelo de Lang de tres sistemas de $\mathrm{R}$ y al modelo de Endler de interacción $\mathrm{S} \times \mathrm{R}$, en los que se basa. Asimismo, se presentan los datos normativos, por sexo y grupo.

Conclusión: Se concluye que el ISRA-B es válido y confiable, mejora su aplicabilidad respecto al ISRA original, conservando sus propiedades psicométricas.
\end{abstract}

(C) 2020 Sociedad Española para el Estudio de la Ansiedad y el Estrés - SEAS. Publicado por Elsevier España, S.L.U. Todos los derechos reservados.

\section{Pyschometric properties of the Brief Inventory of Situations and Responses of Anxiety (ISRA-B)}

\section{A B S T R A C T}

Introduction and objectives: Anxiety is the most studied emotional reaction, which alerts us of the possibility of obtaining a negative result, making it adaptive in the vast majority of cases, although it is also one of the underlying factors of the most prevalent emotional disorders. Therefore, its assessment has generated a lot of research and transfer. The Inventory of Situations and Responses of Anxiety (ISRA) was developed in Spain and it includes 22 situations ( $\mathrm{S}$ ) and 24 responses (R) of anxiety, by which 224 items are formed by crossing them (i.e., «in such Situation, I experience this Reaction»). For more than three decades, it has been widely used in research and practice, and its psychometric properties have been

\footnotetext{
* Autor para correspondencia.

Correo electrónico: rogermn@unizar.es (R. Muñoz-Navarro).
} 
published in different countries. A shorter, 46-item version (ISRA-B), with a $22 \mathrm{~S}$ scale plus another of 24 $\mathrm{R}$ was developed. The same eight facets of anxiety were assessed, showing good psychometric properties as well. However, there are few publications on the psychometric data using Spanish population. The objective of this study was to analyze the psychometric properties of ISRA-B in Spain.

Methods: Three thousand three hundred and forty six volunteers ( $36.4 \%$ men), aged 15 to 94 years (mean: 30.3; SD: 14.6) were included.

Results: The results confirmed excellent internal consistency for the Total (.94) and the Responses subscale (0.91), good for the Situations (0.89), as well as the Physiological (, 86) and Cognitive (0.84) response systems, and acceptable for the Motor ( 0.70$)$. Good test-retest stability was also found after two months (0.78) and good external validity when correlated with the original ISRA $(0.87)$. The results confirm the factor structure of the ISRA-B, adjusted to Lang's triple response system model and to Endler's $\mathrm{S} \times \mathrm{R}$ interaction model, on which it is based. Likewise, the normative data are presented, differentiated by sex and group.

Conclusions: It is concluded that the ISRA-B is a valid and reliable instrument, which improves its applicability compared to the original ISRA by simplifying its format and number of items, while preserving its psychometric properties.

๔ 2020 Sociedad Española para el Estudio de la Ansiedad y el Estrés - SEAS. Published by Elsevier España, S.L.U. All rights reserved.

\section{Introducción}

La ansiedad ha sido ampliamente estudiada como un constructo unidimensional, el rasgo general de ansiedad, evaluado por diferentes instrumentos, destacando el STAI con diecisiete mil citas (García-Batista et al., 2017). Sin embargo, el estudio de la ansiedad en sus diferentes facetas, como reacción emocional en los tres sistemas de respuesta $(R)$ y ante diferentes situaciones $(S)$, ha tenido un buen desarrollo en las últimas décadas, lo que ha permitido crear diferentes instrumentos de evaluación que miden dichas facetas, que resultan útiles para diversos propósitos (Chust-Hernández, Castellano-Rioja, Fernández-García, \& Chust-Torrent, 2019; GarcíaFernández, Inglés, Martínez-Monteagudo, Marzo, \& Estévez, 2011).

El Inventario de Situaciones y Respuestas de Ansiedad, ISRA (Miguel-Tobal y Cano-Vindel, 1986, 2002) fue desarrollado siguiendo el formato situación por respuesta $(\mathrm{S} \times \mathrm{R})$ propuesto por el modelo interactivo de Endler (Cano-Vindel, 1989; Endler y Okada, 1975) e incluye manifestaciones de los tres sistemas de R (cognitivo, fisiológico, motor), propuestos por el modelo multidimensional de la emoción de Lang de 1968 (Martínez-Monteagudo, Inglés, Cano-Vindel, \& García-Fernández, 2012), por lo que permite la evaluación de R concretas Cognitivas (C), Fisiológicas (F) y Motoras $(\mathrm{M})$ de ansiedad ante diferentes $\mathrm{S}$, que se agrupan en cuatro áreas o rasgos específicos: situaciones de Evaluación, Interpersonales, Fóbicas y Cotidianas. El Total (T) de la prueba o nivel general de ansiedad está formado por la suma de las tres subescalas de $\mathrm{R}$ (C, F y M) y puede ser entendido como el rasgo general de ansiedad. Por lo tanto, permite la obtención de ocho medidas o facetas diferentes de la ansiedad, lo que lo ha convertido de hecho en uno de los inventarios más usados en la práctica habitual de los psicólogos en España (Muñiz Fernández y Fernández-Hermida, 2010; Sanz, 2014).

En la investigación, el ISRA ha sido muy utilizado en diferentes países, especialmente en el ámbito de la psicología clínica (Aparicio-García, Fernández-Castilla, Giménez-Páez, Piris-Cava, \& Fernández-Quijano, 2018; Cano-Vindel y Espada Largo, 2003; Entradas Saavedra, Ferreiro Cotorruelo, Folk Madrid, \& Marín Fuste, 1992; González Ordi, 1994; Miguel-Tobal \& Cano-Vindel, 1995; Rodríguez López, González Ordi, Cano Vindel, \& Iruarrizaga Díez, 2007; Solano Pinto y Cano-Vindel, 2012) y la psicología de la salud (Cano-Vindel, Fernández, \& Spielberger, 2012; Cano-Vindel y Fernández Rodríguez, 1999; García-Torres, Alós, \& Castillo-Mayén, 2018; Moreno-Rosset y Martín Díaz, 2009; Nogués Moyano, González Barrón, \& Sifre García, 2000; Pérez Pareja, Borrás
Sansaloni, \& Palmer Pol, 1994; Redondo Delgado, Miguel Tobal, \& Pérez Nieto, 2007; Fernández Rodríguez, 2012), pero también en otros campos de la psicología, como la psicofisiología (Villada, Hidalgo, Almela, \& Salvador, 2016; Villada, Hidalgo Almela, \& Salvador, 2018; Yorio, Pompilio, Leibovich, \& Segura, 1998), psicometría (Camuñas, Cano-Vindel, Pérez-Nieto, \& González-Ordi, 2002; Richaud de Minzi y Sacchi, 1995; Sánchez-López y Dresch, 2008; Terol Cantero et al., 2007), delimitación de constructos (Sanz Fernández, 1991), estudio de procesos cognitivos (Calvo, Avero, Castillo, \& Miguel-Tobal, 2003; Medrano, Muñoz-Navarro, \& CanoVindel, 2016), psicología de las organizaciones (Pérez-Nieto et al., 2001), psicología del deporte (García-Naveira Vaamonde y RuizBarquín, 2015; Miguel Tobal, Navlet Salvatierra, \& Martín Díaz, 2001) o problemas de ansiedad de evaluación (Furlan y SánchezRosas, 2018).

En la validación española $(\mathrm{N}=1,209)$ la prueba original mostró datos favorables para consistencia interna, estabilidad y validez de criterio, con excelentes valores de consistencia interna para $\mathrm{T}$ (.99), los tres sistemas de R (entre .95 y .98) y los cuatro tipos de $S$ (entre .92 y .96); así como buenos valores de correlación test-retest $(\mathrm{N}=101$ ) para $\mathrm{T}(.81)$, sistemas de $\mathrm{R}$ (entre .74 y .78) y $\mathrm{S}$ (entre .74 y .81); y, finalmente, se obtuvieron correlaciones de .69 y .64 entre $\mathrm{T}$ y la Escala de Ansiedad Manifiesta (MAS) de Taylor y el STAI de Spielberger, respectivamente (Cano-Vindel y Miguel-Tobal, 1999; Miguel-Tobal y Cano-Vindel, 2002).

La estructura factorial del ISRA ha sido estudiada tanto para las $24 \mathrm{R}$ como las $22 \mathrm{~S}$, arrojando en ambos casos estructuras compatibles con los modelos teóricos en los que se basa y lejos de un modelo unifactorial de esta reacción emocional. Las $\mathrm{R}$ se explican bien en un modelo de tres factores, que viene a coincidir en los análisis factoriales exploratorios (AFE) con la clasificación racional de ítems en los tres sistemas. A su vez, las $22 \mathrm{~S}$ se vienen agrupando en cuatro factores ya mencionados. En España, con una muestra de 1,209 personas, al analizar las R empleando el método PCA (componentes principales) con rotación oblicua (DQUART), se obtuvo una solución de tres factores (C, F y M) que explicó el 64.7\% de la varianza, con buen ajuste general, lo que apoya la idea de una estructura acorde con el modelo de Lang (Cano-Vindel y Miguel-Tobal, 1999; Miguel-Tobal y Cano-Vindel, 2002). Para las S, en España, con los mismos procedimientos y muestra anteriores, se encontraron cuatro factores (situaciones de evaluación, interpersonales, fóbicas y cotidianas) que explicaron el $67.9 \%$ de la varianza, aunque algunos ítems no encajaron correctamente. Estos cuatro factores agruparon a los cuatro tipos de $\mathrm{S}$ ya mencionados, los cuales guardaron 
similitud con los encontrados por Endler con un inventario diferente y muestran la necesidad de evaluar la ansiedad ante diferentes tipos de $\mathrm{S}$, dando apoyo a la teoría interactiva de la ansiedad de Endler y al concepto de rasgo específico (Cano-Vindel y MiguelTobal, 1999; Miguel-Tobal y Cano-Vindel, 2002).

En diferentes estudios el ISRA ha mostrado una buena capacidad de discriminación entre distintos grupos y patologías, revelando alta sensibilidad para el estudio de las diferencias intergrupo y sobre eficacia de los tratamientos. Se han publicado artículos con una amplia muestra de poblaciones clínicas, entre ellas: cefaleas, trastornos de piel, síndrome premenstrual, hipertensión, diferentes trastornos psicofisiológicos, diversos trastornos de ansiedad, trastornos del estado de ánimo, delincuencia juvenil, juego patológico (Cano-Vindel y Miguel-Tobal, 1999). En todos estos casos el ISRA ha mostrado una alta capacidad de discriminación, manifestando diferencias altamente significativas y tamaños del efecto altos o medios entre los diferentes grupos y patologías evaluados (García Batista y Cano-Vindel, 2014), así como a la hora de demostrar la eficacia de los tratamientos (Martínez-Monteagudo et al., 2012; Nieto Fernández y Barraca Mairal, 2017). Incluso en la controvertida diferenciación entre los constructos de ansiedad y depresión por medio de autoinformes, Sanz Fernández (1991, p. 170) señaló que «en relación a la diferenciación psicométrica entre ansiedad y depresión, las subescalas de rasgos específicos de ansiedad del ISRA (tipos de situaciones) se configuran como instrumentos de elección a la hora de discriminar entre sujetos ansiosos y depresivos frente a las tan utilizadas medidas de rasgo general de ansiedad»; a lo que añade que "de hecho, la inclusión de medidas de rasgo específico de ansiedad permite que la depresión aparezca en los análisis factoriales como un constructo relativamente independiente y discernible de la ansiedad».

Como ya se ha señalado, el ISRA ha sido traducido a varios idiomas y validado para distintas poblaciones (Cano-Vindel y Miguel-Tobal, 1999), existiendo versiones disponibles para diferentes países, incluidos Estados Unidos, Brasil, Portugal, Holanda, Alemania, Francia, Italia, Finlandia, Suecia, Polonia, Argentina, Cuba, México, Costa Rica y República Dominicana (AmorimGaudêncio, Roustan, \& Sirgo, 2004; García-Batista, Cano-Vindel, \& Herrera-Martínez, 2015; García Batista y Cano-Vindel, 2014; González-Ramírez, Quezada-Berumen, del, Díaz-Rodríguez, \& Cano-Vindel, 2014; Jurado Solorzano, 2010; Leibovich de Figueroa, 2001; Losiak, 2005; Medrano et al., 2016; Moretti, Galeazzi, MiguelTobal, \& Cano-Vindel, 2006; Rodríguez Martín, Molerio Pérez, Martínez Rodríguez, González Paneca, \& Navarro Otero, 2014). En los estudios de estabilidad y validación criterial con las versiones brasileña $(\mathrm{N}=101)$, norteamericana de habla inglesa $(\mathrm{N}=40) \mathrm{o}$ dominicana $(\mathrm{N}=993)$ se obtuvieron valores muy similares a los encontrados en España sobre estabilidad test-retest y validez de criterio con el STAI (Cano-Vindel y Miguel-Tobal, 1999; García Batista y Cano-Vindel, 2014). El AFE de las R mostró resultados similares a los de la validación española en la versión norteamericana $(\mathrm{N}=155)$, en la versión brasileña $(\mathrm{N}=1,136)$, en República Dominicana $(N=1,074)$ y con la versión portuguesa $(N=409)$. En cuanto al análisis de las $\mathrm{S}$, en Brasil, con la misma muestra, se obtuvo una réplica bastante similar de los resultados de España, encontrando un $73.4 \%$ de varianza explicada en dicha solución de cuatro factores. En Portugal, se encontró un modelo similar de cuatro factores que explicó el 71\% de la varianza. En USA, encontraron que dos tipos de situaciones muy relacionadas, evaluación e interpersonal, se agrupaban en un solo factor (Cano-Vindel y Miguel-Tobal, 1999).

Aunque es una ventaja la gran cantidad de información que puede suministrar el ISRA sobre distintas facetas de la ansiedad, una de sus limitaciones para su aplicabilidad es su excesivo número de ítems (Medrano, Moretti, Muñoz-Navarro, \& Cano-Vindel, 2017). Un primer intento por conseguir una versión abreviada de tan solo $17 \mathrm{R}$ de ansiedad, agrupadas en los tres sistemas de respuesta, obtuvo buenas propiedades psicométricas pero se prescindió de evaluar las S, se modificaron las opciones de respuesta y no generó mucha investigación (Martínez Sánchez et al., 1995). Sin embargo, cuando se han mantenido las mismas $22 \mathrm{~S}$ y las $24 \mathrm{R}$ de ansiedad originales, con idéntica escala de respuesta, y simplemente se ha sustituido el formato interactivo $\mathrm{S} \times \mathrm{R}$ por dos escalas diferentes $\mathrm{S}$ más $\mathrm{R}$, el número de ítems se ha reducido desde 224 a 46, pero se ha conservado la cantidad de información que proporciona, es decir las ocho subescalas originales, y esta nueva versión (ISRA-B) sí ha generado más investigación en diferentes países (GonzálezRamírez et al., 2014; Medrano et al., 2017).

Existen menos evidencias sobre las propiedades psicométricas de esta versión abreviada (ISRA-B) que de la versión original. Aunque el ISRA-B ha mostrado buenas propiedades psicométricas en la población de México (González-Ramírez et al., 2014) y de Argentina (Medrano et al., 2017, 2016), no se han publicado artículos describiendo de forma monográfica las propiedades psicométricas del ISRA-B en la población española, a pesar de ser citadas algunas de ellas en algunas publicaciones que no estaban centradas en este objetivo (Cano-Vindel y Macías, 2002; Macías-Delgado et al., 2014; Medrano et al., 2016; Moreno-Rosset y Martín Díaz, 2009; Nieto Fernández y Barraca Mairal, 2017). Por ello, el propósito de este estudio es analizar las propiedades psicométricas del ISRA-B en población española.

\section{Método}

\section{Participantes}

Se incluyeron los datos de 3,346 voluntarios (36.4\% hombres), con edades de 15 a 94 años (media: 30.3; DE: 14.6). Se usó una amplia variedad de grupos demográficos con el objeto de evaluar si los ítems del ISRA-B presentan un comportamiento robusto en la población general y en diferentes grupos: población general de distintas áreas geográficas y variadas características personales, captados por procedimientos de bola de nieve $(n=2,025)$, estudiantes de primer curso de psicología $(n=773)$, muestra clínica con diferentes trastornos de ansiedad que demandaban tratamiento $(n=290)$ y personas con problemas de salud crónicos recogidos por procesos de bola de nieve $(\mathrm{n}=258)$, de los cuales había pacientes con cáncer $(n=120)$, artritis reumatoide $(n=40)$, Parkinson $(n=43)$ y psicosomáticos $(n=45)$. Los participantes fueron seleccionados mediante un muestreo no probabilístico de tipo accidental, colaboraron de manera voluntaria y proporcionaron su consentimiento informado. Además, se garantizó el carácter anónimo y confidencial de sus datos.

\section{Instrumentos}

ISRA. Como ya se ha descrito, el ISRA (Miguel-Tobal y Cano-Vindel, 1986, 2002) es un instrumento con formato interactivo $\mathrm{S} \times \mathrm{R}$, que incluye $24 \mathrm{R}$ de ansiedad (7 de tipo $\mathrm{C}, 10$ de tipo $\mathrm{F}$ y 7 de tipo M), así como $22 \mathrm{~S}$ que pueden ser agrupadas en cuatro tipos. De los cruces $\mathrm{S}$ (filas) $\times \mathrm{R}$ (columnas) resultan un conjunto de 224 ítems, o cuadros blancos a responder, tras anular los cruces que se han desechado (cuadros negros, a los que no hay que responder), por diferentes motivos: excesivo número de ítems, eliminación de ítems con menor poder de discriminación, o con menor posibilidad de ocurrencia, etc. La selección de los ítems se llevó a cabo utilizando una triple metodología racional, empírica y factorial (Miguel-Tobal y Cano-Vindel, 1986). El ISRA permite la evaluación de los tres sistemas de $\mathrm{R}$ de ansiedad por separado, la suma de los tres ( $\mathrm{T}$ ), así como de la ansiedad asociada a cuatro áreas situacionales o rasgos específicos. Estos rasgos específicos de ansiedad son similares a los obtenidos por Endler en el marco del modelo 
interactivo de ansiedad. Se refieren a diferencias individuales ligadas a áreas situacionales o tendencias de los individuos a manifestar diferente grado de ansiedad en distintos tipos de situaciones (CanoVindel, 1989). La estabilidad test-retest al cabo de un mes $(\mathrm{N}=101)$ fue .81 . La consistencia interna alcanzada fue excelente $(\mathrm{N}=1,209)$, con valores que oscilan entre .92 y .99, para las ocho escalas en la versión española. La validez convergente mostró una correlación de $.64(\mathrm{~N}=383)$ entre el total del ISRA y el STAI de Spielberger (Miguel-Tobal y Cano-Vindel, 1986, 2002).

ISRA-B. Inventario de Situaciones y Respuestas de Ansiedad (versión breve) (Miguel-Tobal y Cano-Vindel, 2002). Esta versión abreviada se desarrolló a partir de las $\mathrm{R}$ y $\mathrm{S}$ del ISRA original (Miguel-Tobal y Cano-Vindel, 1986; 2002). Los 46 ítems evalúan la frecuencia con la que se experimenta ansiedad, de acuerdo a una escala tipo Likert que va desde casi nunca (0) hasta casi siempre (4) para la escala $\mathrm{R}$ y desde nada (0) a muchísimo (4) para la escala $\mathrm{S}$. Los primeros 24 ítems evalúan $\mathrm{R}$ de ansiedad (escala $\mathrm{R}$ ) a nivel C (respuestas 1 a 7 , con ítems como "me preocupo demasiado», p.ej.), F (los siguientes 10 ítems, como «me tiemblan las manos o las piernas», p.ej.) y M (respuestas 18 a 24, 7 ítems, «me muevo y hago cosas sin una finalidad concreta», p.ej.). Permiten obtener una puntuación de ansiedad autoinformada en el total de R, más otras tres en cada uno de los sistemas. Los restantes 22 reactivos evalúan la ansiedad ante situaciones concretas o escala S. La mayoría pueden agruparse en cuatro tipos de situaciones ansiógenas, más concretamente sobre Situaciones de Evaluación ( 7 ítems, "ante un examen o si voy a ser entrevistado para un trabajo importante», p.ej.), situaciones Interpersonales ( 5 ítems, «cuando tengo que asistir a una reunión social o conocer gente nueva», p.ej.), Fóbicas (5 ítems, "cuando tengo que viajar en un avión o barco», p.ej.) y de la vida Cotidiana (5 ítems, "a la hora de dormir», p.ej.). Permiten obtener una puntuación en el total de $S$, más otras cuatro para cada tipo de situaciones. La puntuación $T$ es la suma de $R$ más S. En trabajos previos se ha encontrado que el ISRA-B inicialmente mostró buena consistencia interna para el Total de los 46 ítems de la escala, .94, para la subescala $\mathrm{R}$ de .90 (C .83, F .86 y M .69) y .89 para la subescala S (Evaluación .81, Interpersonales .63, Fóbicas .78 y Cotidianas .61) (González-Ramírez et al., 2014).

STAI. El Inventario de Ansiedad Estado-Rasgo (State-Trait Anxiety Inventory), evalúa la ansiedad Estado y el Rasgo mediante 20 ítems para cada escala, con opciones de respuesta tipo Likert de cuatro alternativas. En el caso de la ansiedad Estado, las opciones van de 0 (Nada) a 3 (Mucho), mientras que en la ansiedad Rasgo desde 0 (Casi nunca) a 3 (Casi siempre). En ambas escalas, un porcentaje de preguntas están invertidas y evalúan bienestar o ausencia de ansiedad, mientras que el resto de los reactivos se refieren a la presencia de ansiedad. La puntuación total se obtiene mediante la suma de los reactivos tras la inversión de los que están redactados en positivo. Sus propiedades psicométricas son bien conocidas (Spielberger, Gorsuch, \& Lushene, 2010).

ASI-3. Se usó la versión española (Sandín, Valiente, Chorot, \& Santed Germán, 2007) del Índice de Sensibilidad a la Ansiedad, versión 3 (ASI-3). Consta de 18 ítems que evalúan el grado en que la persona suele experimentar cada uno de los enunciados de acuerdo a una escala tipo Likert, que va desde nada o casi nada (0), hasta muchísimo (4). Los ítems hacen referencia a reacciones de miedo/ansiedad ante la experiencia de síntomas de tipo físico, síntomas de descontrol cognitivo y síntomas socialmente observables. Incluye tres subescalas de sensibilidad a la ansiedad (física, cognitiva y social), cada una de las cuales está constituida por seis ítems. La consistencia interna de la versión española varía de .83 a .91 en las diferentes subescalas, presenta adecuada estabilidad testretest (correlaciones entre .83 y .85 para las diferentes subescalas) y confirmación de su dimensionalidad teórica a través del análisis factorial exploratorio (Sandín et al., 2007).
MAS. Escala de Ansiedad Manifiesta (Taylor, 1953). Esta escala fue una de las primeras en medir la ansiedad como un constructo unidimensional. Fue construida a partir de los ítems que medían ansiedad del MMPI, por lo que se centra en aspectos clínicos y de la personalidad. Consta de 50 ítems que se responde como falso y verdadero. Ha sido usada en múltiples ocasiones para calcular la validez convergente de instrumentos más modernos, incluido el ISRA.

\section{Análisis de datos}

Se realizaron diferentes análisis exploratorios de datos, sobre tendencia central, dispersión, asimetría y apuntamiento (curtosis), así como pruebas de normalidad uni y multivariante. Para la validez de constructo se analizaron diversos modelos de estructura, tanto de la escala $\mathrm{R}$ como de la $\mathrm{S}$, mediante análisis factorial exploratorio (AFE) y confirmatorio (AFC). Para la estimación de modelos AFE se emplearon de forma previa el test de esfericidad de Bartlett y el índice KMO, así como el criterio de Kaiser para la extracción de factores con rotación Promax. Además, se realizó un AFC usando el método ML de máxima verosimilud, mediante AMOS versión 25 , para evaluar el ajuste de los datos obtenidos en la escala R al modelo tridimensional propuesto, frente al modelo unitario de la ansiedad. También se llevó a cabo otro AFC con el método ML para la escala S, con el fin de analizar las dimensiones de las situaciones, llamadas también rasgos específicos de ansiedad. Se utilizaron varios índices absolutos y relativos o comparativos, en concreto: el estadístico chicuadrado $\left(\chi^{2}\right)$, el error cuadrático medio de aproximación (RMSEA, Root Mean Square Error of Approximation), su intervalo de confianza (IC 90\%), el índice de bondad de ajuste (GFI), el índice ajustado de bondad de ajuste (AGFI), el índice de ajuste no normalizado o índice de Tucker-Lewis (NNFI o TLI) y el índice de ajuste comparativo (CFI, Comparative Fit Index). También se utilizaron algunos índices de ajuste de la parsimonia, en concreto la razón de parsimonia (PRATIO), el índice de ajuste comparativo de la parsimonia (PCFI), el índice de ajuste normalizado de parsimonia (PNFI) y el índice de criterio de información de Akaike (AIC). Para interpretar dichos índices, se consideraron los valores críticos recomendados por la literatura, que propone como aceptables valores superiores a .90 para los índices GFI, AGFI, TLI, CFI y PRATIO, valores superiores a .80 para PCFI y PNFI, e inferiores a .08 para el RMSEA, así como el menor valor posible para el AIC (Byrne, 2001; Hu y Bentler, 1999).

Al analizar dicha estructura interna o de constructo, tanto para $\mathrm{R}$ como para $\mathrm{S}$, con el fin de establecer una estrategia de comparación de modelos, se comenzó por la estimación de un modelo unifactorial, que defiende el constructo unitario de la ansiedad, ya sea el concepto de reacción emocional como un constructo unitario de $\mathrm{R}$, bien el concepto de rasgo general de ansiedad, independiente de las S. Sin embargo, el inventario original se construyó sobre una estructura latente de tres componentes para las $\mathrm{R}$ de ansiedad, con los siete primeros ítems para el C, los 10 siguientes para $\mathrm{F}$ y los siete últimos para M. Esta misma estructura y número de ítems se ha conservado en el ISRA-B. Por lo que se refiere a la escala $S$, ambas versiones del ISRA incluyeron 22 reactivos, con los que se suelen enfrentar las personas con cierta frecuencia, y en su construcción se partió del supuesto de la existencia de varios tipos de situaciones o rasgos específicos de ansiedad, suponiendo que los rasgos específicos explicarán más varianza y tendrán más poder predictivo sobre el nivel de ansiedad en una determinada situación que el rasgo general de ansiedad (Cano-Vindel, 1989). Además, en ambos casos, se analizó el ajuste de un modelo bifactor, donde se propone un factor general que representa la ansiedad como reacción global más los tres factores específicos de R; y en el caso de las $\mathrm{S}$, el rasgo general de ansiedad ante cualquier situación, más los cuatro factores específicos de $\mathrm{S}$ (rasgos específicos de ansiedad). 
Tabla 1

Análisis Factorial Exploratorio de la escala R (respuestas) del ISRA-B ( $\mathrm{N}=3,346)$

\begin{tabular}{|c|c|c|c|c|c|}
\hline & & & & & Componente \\
\hline & 1 & 2 & 3 & Escala & Ítems \\
\hline r15 & .757 & & & $\mathrm{~F}$ & Siento náuseas o mareo \\
\hline r14 & .732 & & & $\mathrm{~F}$ & Me falta el aire y mi respiración es agitada \\
\hline r8 & .656 & & & $\mathrm{~F}$ & Siento molestias en el estómago \\
\hline r13 & .647 & & & $\mathrm{~F}$ & Tengo palpitaciones, el corazón me late muy deprisa \\
\hline r11 & .634 & & & $\mathrm{~F}$ & Me duele la cabeza \\
\hline r12 & .618 & & & $\mathrm{~F}$ & Mi cuerpo está en tensión \\
\hline r16 & .584 & & & $\mathrm{~F}$ & Se me seca la boca y tengo dificultades para tragar \\
\hline r17 & .539 & & & $\mathrm{~F}$ & Tengo escalofríos y tirito aunque no haga mucho frío \\
\hline $\mathrm{r} 10$ & .537 & & & $\mathrm{~F}$ & Me tiemblan las manos o las piernas \\
\hline r9 & .478 & & & $\mathrm{~F}$ & Me sudan las manos u otra parte del cuerpo hasta en días fríos \\
\hline r3 & & .742 & & $\mathrm{C}$ & Me siento inseguro \\
\hline $\mathrm{r} 4$ & & .736 & & $\mathrm{C}$ & Doy demasiadas vueltas a las cosas sin llegar a decidirme \\
\hline r1 & & .735 & & $\mathrm{C}$ & Me preocupo fácilmente \\
\hline r2 & & .702 & & $\mathrm{C}$ & Tengo pensamientos o sentimientos negativos sobre mí, tales como «inferior» a los demás, "torpe», etc. \\
\hline r5 & & .623 & & $\mathrm{C}$ & Siento miedo \\
\hline r18 & & .510 & & M & Lloro con facilidad \\
\hline r7 & & .495 & .427 & $\mathrm{C}$ & Pienso que la gente se dará cuenta de mis problemas o de la torpeza de mis actos \\
\hline r6 & & .402 & .310 & $\mathrm{C}$ & Me cuesta concentrarme \\
\hline r24 & & & .701 & M & Tartamudeo o tengo otras dificultades de expresión verbal \\
\hline r23 & & & .700 & M & Quedo paralizado o mis movimientos son torpes \\
\hline r22 & & & .700 & M & Me muevo y hago cosas sin una finalidad concreta \\
\hline r19 & & & .461 & M & Realizo movimientos repetitivos con alguna parte de mi cuerpo (rascarme, tocarme, movimientos rítmicos con pies o manos, etc.) \\
\hline r20 & & & .417 & M & Fumo, como o bebo demasiado \\
\hline r21 & & & .369 & M & Trato de evitar o rehuir las situaciones que pueden producir tensión \\
\hline
\end{tabular}

Método de extracción: análisis de componentes principales. Método de rotación: Promax con normalización Kaiser. Se han eliminado los pesos inferiores .30. Notas: r1 hasta r24 son las respuestas de ansiedad incluidas en la escala R. C: Sistema de respuesta Cognitivo, F: Fisiológico, M: Motor o conductual.

La fiabilidad de las escalas del cuestionario se analizó calculando su consistencia interna mediante el índice alfa de Cronbach, fijando el punto de corte por encima del .70 recomendado (Nunnally y Bernstein, 1994). Para evaluar la estabilidad se correlacionaron las puntuaciones del ISRA-B con un lapso de un mes entre las administraciones.

La validez convergente o concurrente fue estudiada mediante análisis de correlación entre las escalas y variables externas, otros constructos con los que teóricamente se encuentran relacionadas, medidos por instrumentos con buenas y conocidas propiedades psicométricas.

Para estudiar la validez discriminante del ISRA- B se analizaron las curvas receiver operating characteristics (ROC). Se calculó el valor estimado para el área y su IC 95\%, cuyo límite inferior debe estar siempre por encima del $80 \%$. Además, se estimaron los puntos de corte que pueden arrojar un mejor balance entre una buena sensibilidad y especificidad para diferenciar el grupo clínico del grupo de población general. Adicionalmente, para estudiar la capacidad de discriminación se analizaron las diferencias en ansiedad entre los grupos estudiados.

Finalmente se calcularon los baremos de la población general para mujeres y para hombres, con centiles y cuartiles, en todas las escalas del ISRA-B.

\section{Resultados}

\section{Evidencias de estructura interna}

Todos los ítems presentaron una distribución cercana a la normalidad con valores de asimetría y curtosis inferiores a \pm 2 . No obstante, el método de estimación de máxima verosimilitud empleado en los análisis más importantes es bastante robusto ante tal incumplimiento y permite obtener una estimación razonable incluso en estas condiciones.

El análisis factorial exploratorio (AFE) de la escala R del ISRAB con extracción por componentes principales y rotación Promax, forzando a tres factores (habría cuatro con valor propio mayor que 1 ), obtuvo una varianza explicada del $46.4 \%$, frente al $33.4 \%$ que explicaría un único factor. La medida Kaiser-Meyer-Olkin de adecuación de muestreo dio un valor muy bueno de $.943 \mathrm{y}$ el valor de ji-cuadrado en la prueba de esfericidad de Bartlett arrojó un valor de 28,058.2 (g.l.: 276; $p<.000$ ). En la solución de tres factores, el primero agrupó a las $10 \mathrm{R}$ Fisiológicas (ítems 8 al 17), con pesos entre .757 y .478 (Tabla 1 ). Ningún otro ítem tuvo un peso superior a .30 en este factor, por lo que representa al sistema $F$. En el segundo factor se encontraron las 7 R Cognitivas (ítems 1 al 7), con pesos entre .742 y .402 . Hubo una excepción, al sumarse el ítem r18 de la escala M ( «Lloro con facilidad», con peso .510). En el tercer factor se mostraron los otros seis ítems de la escala M (ítems 18 al 24), con pesos entre.701 y.369; pero los ítems Cognitivos 6 y 7 («Pienso que la gente se dará cuenta de mis problemas o de la torpeza de mis actos»; "Me cuesta concentrarme») tuvieron también pesos en este factor (M o conductual observable).

El análisis factorial confirmatorio (AFC) por el método ML de las $\mathrm{R}$ mostró que los datos de este estudio no se ajustaron al modelo unitario de la ansiedad, como puede verse en la Tabla 2. El inventario original se construyó sobre una estructura latente con tres componentes: C, F, y M, que se respeta en la versión abreviada. Una vez sometido a estimación, el ajuste del modelo trifactorial fue sustancialmente mejor que el obtenido por el modelo unifactorial. El valor de ji-cuadrado, $\chi^{2}$, fue significativo $(3,146.83 ; p<.0001$ ), y la función penalizadora fue superior a tres (12.54), y por tanto, indicadores de mal ajuste, aunque con una muestra tan grande son esperables estos resultados, al menos en el primero de estos índices de ajuste. Por su parte, el valor del RMSEA (.059; IC 90\%: .057; .061) tampoco confirmó un ajuste óptimo $(<.05)$, pero sí aceptable [P(RMSEA $)<.05=.001]$, y lo mismo ocurrió con el RMR (.061), claramente por encima de .05 , pero por debajo de .08 . El índice de bondad de ajuste, GFI, fue aceptable (.920); el índice ajustado de bondad de ajuste, AGFI, también (.904); y el índice de ajuste comparativo, CFI, prácticamente también (.896). A su vez, los índices de ajuste de la parsimonia fueron aceptables para la razón de parsimonia, PRATIO (.909), el índice de ajuste comparativo de parsimonia, PCFI (.815) y el índice de ajuste normalizado de parsimonia, PNFI, (.808). El índice AIC de este modelo de tres factores mostró un valor $(3,292.83)$ mucho menor que el del modelo unitario (7,205.20). Estos datos 
Tabla 2

Índices de Ajuste del Modelo de Respuestas de Ansiedad

\begin{tabular}{|c|c|c|c|c|c|c|c|c|c|c|c|}
\hline & Chi-cuadrado & RMSEA & GFI & AGFI & $\mathrm{CFI}$ & TLI & NFI & PRATIO & PCFI & PNFI & AIC \\
\hline Bueno & $\mathrm{p}>.05$ & $<.05$ & $\geq .95$ & $\geq .95$ & $\geq .90$ & $\geq .95$ & $\geq .95$ & $\geq .95$ & $\geq .80$ & $\geq .70$ & el menor \\
\hline $\begin{array}{l}\text { Aceptable } \\
\text { Modelos }\end{array}$ & $\mathrm{p}>.06$ & $<.08$ & $\geq .90$ & $\geq .90$ & $\geq .90$ & $\geq .90$ & $\geq .90$ & $\geq .90$ & $\geq .70$ & $\geq .60$ & el menor \\
\hline Un factor & 7,$107 ; 2.000$ & .086 & .807 & .790 & .755 & .754 & .747 & .996 & .752 & .745 & $7,205.20$ \\
\hline Tres factores & 3,$146 ; 83.000$ & .059 & .920 & .904 & .896 & .886 & .888 & .909 & .815 & .808 & $3,292.83$ \\
\hline Bifactor & 3,$088 ; 43.000$ & .058 & .922 & .907 & .898 & .888 & .890 & .909 & .817 & .810 & $3,234.43$ \\
\hline
\end{tabular}

sobre ajuste aceptable, pero no óptimo, junto a los obtenidos tras el análisis de la estructura exploratoria (AFE) indicaron que todos los ítems obtuvieron pesos significativos, aunque algunos presentaron ciertos problemas de covariabilidad. Si bien se ajustan a los criterios comúnmente aceptados, podrían eliminarse algunos ítems con pesos más bajos, como por ejemplo r18, r6 y r7, hasta conseguir un ajuste óptimo y una escala más reducida. Por último, los resultados sobre el ajuste del modelo bifactor de las R fueron ligeramente mejores que los del modelo de los tres factores (AIC: 3,234.43 vs. 3,292.83), no observándose una mejora sustancial (Tabla 2).

El análisis factorial exploratorio (AFE) de la escala S del ISRA-B con extracción por ML, máxima verosimilitud, y rotación Promax, corrobora en líneas generales la solución de cuatro factores definida previamente, que se maneja hasta ahora en el manual del ISRA y que se ha mantenido para el ISRA-B, añadiendo una situación en cada fórmula (la S9 en F1, la S6 en F2, la S20 en F3 y la S2 en $\mathrm{F} 4$ ), con el fin de aumentar la consistencia interna de los cuatro rasgos específicos del ISRA-B. En el factor, de ansiedad en situaciones de Evaluación, saturaron las siete situaciones de la fórmula de corrección del ISRA-B (F1 = S1 + S4 + S8 + S9 + S10 + S11 + S13). En el segundo factor, de ansiedad ante situaciones Cotidianas, que en el Manual del ISRA es llamado F4, tuvieron pesos también las cuatro situaciones de la fórmula $(\mathrm{F} 4=\mathrm{S} 2+\mathrm{S} 5+\mathrm{S} 21+\mathrm{S} 22)$. En el tercer factor, de ansiedad ante situaciones Fóbicas, que en el manual del ISRA se denomina F3, saturaron las cinco de la fórmula $(\mathrm{F} 3=\mathrm{S} 12+\mathrm{S} 14+\mathrm{S} 17+\mathrm{S} 19+\mathrm{S} 20)$. Finalmente, en el cuarto factor de esta solución, denominado Interpersonal, identificado como F2, también estuvo formado por las cuatro situaciones de su fórmula de corrección (F2 = S6 + S7 + S15 + S18). Las situaciones S3 y S16, que hasta ahora no se incluían en ninguna de las fórmulas, en esta solución factorial saturaron en el factor de situaciones Cotidianas. Por otro lado, las situaciones S4 y S6 presentaron saturaciones en dos factores (Tabla 3).

El análisis factorial confirmatorio (AFC) por el método ML de las $22 \mathrm{~S}$ mostró que los datos no se ajustaron al modelo unitario, sino que apoyaron más el modelo de cuatro factores situacionales. Para mejorar el ajuste, se incluyeron S3 y S16 en el grupo de situaciones Cotidianas (F4 = S2 + S3 + S5 + S16 + S21 + S22), tanto para la reespecificación del modelo de cuatro factores como para el bifactor. Los resultados del modelo bifactor de las $\mathrm{S}$ fueron ligeramente mejores que los del modelo de cuatro factores reespecificado o modelo de cuatro factores bis (AIC: $3,132.54$ vs. 3,544.67), aunque no se observó una mejora sustancial (Tabla 4).

\section{Análisis de confiabilidad: consistencia interna y estabilidad}

La consistencia interna alcanzó los valores de $.94, .91$ y .89 para las escalas $\mathrm{T}, \mathrm{R}$ y $\mathrm{S}$, respectivamente. La obtenida por las demás escalas puede verse en la Tabla 5. En definitiva, el ISRA-B presentó una excelente consistencia interna, tanto para los 46 ítems del Total como los 24 de R, buena para los 22 de S y los siete de Evaluación, así como para $\mathrm{C}$ y F, aceptable para M, Interpersonal y Cotidianas, pero cuestionable para Fóbicas, aunque próximo a .70 (.69).

La estabilidad test-retest (correlación de Pearson, $\mathrm{r}$ ), al cabo de 1 mes en una submuestra de población general $(\mathrm{N}=201)$, fue de .78 para la escala $T$, de .73 para $\mathrm{R}$ y .73 para $S$. Para los tres sistemas de respuesta y áreas situacionales se alcanzaron valores similares (Tabla 5).

\section{Evidencias de validez con fuentes externas}

En cuanto al grado de validez de la versión abreviada con la extensa, las correlaciones entre las puntuaciones obtenidas en el ISRA-B de 46 ítems y las alcanzadas en la versión original de 224 ítems $(\mathrm{N}=63)$ pueden verse en la Tabla 6 , siendo de .87 para el Total de ambos instrumentos, así como .84 para el total de $\mathrm{R}$ y .83 para el total de S. Para los tres sistemas de R y las cuatro áreas situacionales se obtuvieron valores en torno a .80 .

Otras pruebas sobre la validez concurrente del ISRA-B, las encontramos en las correlaciones entre el ISRA-B y el ASI-3 en una submuestra de población general $(\mathrm{N}=936)$. Como puede verse en la Tabla 5, se encontró una correlación de .61 entre el Total del ISRA-B y el total del ASI, versión 3.

Al estudiar la validez discriminante para los hombres, el análisis mediante curva ROC de la escala T del ISRA-B (46 ítems) mostró que tiene una excelente capacidad, pues alcanzó un valor estimado para el área del 92.0\% (IC 95\%: 89.3; 94.7), siempre por encima del $80 \%$, con una sensibilidad del $88.0 \%$ y una especificidad del $16.8 \%$ ( 1 especificidad: $83.2 \%$ ) para diferenciar el grupo clínico del grupo de población general, mediante el punto de corte óptimo que definió con mayor exactitud el cribado diagnóstico, que fueron 69 puntos.

Al estudiar la validez discriminante para las mujeres, el análisis mediante curva ROC de la escala T del ISRA-B ( 46 ítems) mostró que tiene una buena capacidad, alcanzando un valor estimado para el área del 86.9\% (IC 95\%: 84.0; 89.7), con una sensibilidad del 84.4\% $\mathrm{y}$ una especificidad del 31.9\% (1-especificidad: $68.1 \%$ ) para diferenciar ambos grupos, clínico y población general, mediante una puntuación de 74 puntos.

La capacidad de discriminación del ISRA-B resultó buena mostrando diferencias significativas en ansiedad entre los grupos estudiados. Así, en la Tabla 7 pueden verse las medias, error estándar, IC 95\%, del Total ISRA-B, en hombres y mujeres de la población general y clínica, con trastorno de pánico y agorafobia. Como puede verse las medias de hombres y mujeres difieren dentro del mismo grupo y sus IC no se solapan. Las medias son siempre más altas en mujeres que en varones. A su vez, las diferencias intergrupo son mucho más pronunciadas. Se encontró un tamaño del efecto grande (eta cuadrado, $\eta^{2}:$.165; d de Cohen: .89) para el factor grupo, pequeño para el sexo (eta cuadrado, $\eta^{2}$ : .027; d de Cohen: .33) y nulo para la interacción.

En la Tabla 8 se muestran el mismo tipo de datos sobre capacidad de discriminación del ISRA-B entre los distintos grupos del estudio $(\mathrm{N}=3,336)$ por sexo. Se encontró un tamaño del efecto grande (eta cuadrado, $\eta^{2}:$.247; d de Cohen: 1.15) para el factor grupo, pequeño para el sexo (eta cuadrado, $\eta^{2}: .012$; d de Cohen: .22) y nulo para la interacción.

\section{Desarrollo de baremos para puntuaciones del ISRA-B}

Los baremos de población general para mujeres y varones pueden verse en las Tablas 9 y 10, respectivamente. En ellas las puntuaciones directas de todas las escalas del ISRA-B pueden ser 
Tabla 3

Análisis Factorial Exploratorio de la escala S (situaciones) del ISRA-B $(\mathrm{N}=3,346)$

\begin{tabular}{|c|c|c|c|c|c|c|}
\hline & \multicolumn{4}{|c|}{ Factor } & \multirow[t]{2}{*}{ Escala } & \multirow[t]{2}{*}{ Ítem } \\
\hline & 1 & 2 & 3 & 4 & & \\
\hline s9 & .668 & & & & Evaluación & $\begin{array}{l}\text { 9. Cuando soy observado o mi trabajo es supervisado, cuando recibo críticas, o siempre que pueda ser } \\
\text { evaluado negativamente }\end{array}$ \\
\hline s11 & .604 & & & & Evaluación & $\begin{array}{l}\text { 11. Cuando pienso en experiencias recientes en las que me he sentido ridículo, tímido, humillado, solo o } \\
\text { rechazado }\end{array}$ \\
\hline s10 & .601 & & & & Evaluación & 10. Si tengo que hablar en público \\
\hline s13 & .486 & & & & Evaluación & 13. Después de haber cometido algún error \\
\hline s1 & .472 & & & & Evaluación & 1. Ante un examen en el que me juego mucho, o si voy a ser entrevistado para un trabajo importante \\
\hline s8 & .380 & & & & Evaluación & 8. Cuando alguien me molesta o cuando discuto \\
\hline s5 & & .621 & & & Cotidianas & 5. En mi trabajo o cuando estudio \\
\hline s3 & & .586 & & & Cotidianas & 3. Cuando pienso en las muchas cosas que tengo que hacer \\
\hline s4 & .370 & .520 & & & Evaluación & 4. A la hora de tomar una decisión o resolver un problema difícil \\
\hline s16 & & .480 & & & Cotidianas & 16. Cuando pienso en mi futuro o en dificultades y problemas futuros \\
\hline$s 22$ & & .438 & & & Cotidianas & 22. A la hora de dormir \\
\hline s2 & & .421 & & & Cotidianas & 2. Cuando voy a llegar tarde a una cita \\
\hline s21 & & .409 & & & Cotidianas & 21. Por nada en concreto \\
\hline s6 & & .340 & & .310 & Interpersonal & 6. Cuando espero a alguien en un lugar concurrido \\
\hline s19 & & & .718 & & Fóbicas & 19. En lugares altos, o ante aguas profundas \\
\hline s12 & & & .629 & & Fóbicas & 12. Cuando tengo que viajar en avión o en barco \\
\hline s17 & & & .518 & & Fóbicas & 17. En medio de multitudes o en espacios cerrados \\
\hline s14 & & & .413 & & Fóbicas & 14. Ante la consulta del dentista, las inyecciones, las heridas o la sangre \\
\hline s20 & & & .383 & & Fóbicas & 20. Al observar escenas violentas \\
\hline s15 & & & & .803 & Interpersonal & 15. Cuando voy a una cita con una persona del otro sexo \\
\hline s7 & & & & .705 & Interpersonal & 7. Si una persona del otro sexo está muy cerca de mí, rozándome, o si estoy en una situación sexual íntima \\
\hline s18 & & & & .369 & Interpersonal & 18. Cuando tengo que asistir a una reunión social o conocer gente nueva \\
\hline
\end{tabular}

Método de extracción: Máxima Verosimilitud. Método de rotación: Promax con normalización Kaiser. Se han eliminado los pesos inferiores .30.

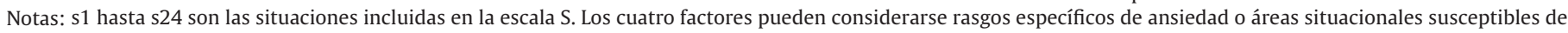
provocar ansiedad: Ansiedad ante Situaciones de Evaluación (test o prueba); Interpersonales o Sociales; Fóbicas y Cotidianas.

Tabla 4

Índices de Ajuste del Modelo de Situaciones de Ansiedad

\begin{tabular}{|c|c|c|c|c|c|c|c|c|c|c|c|}
\hline & Chi-cuadrado & RMSEA & GFI & AGFI & CFI & TLI & NFI & PRATIO & PCFI & PNFI & AIC \\
\hline Bueno & $\mathrm{p}>.05$ & $<.05$ & $\geq .95$ & $\geq .95$ & $\geq .95$ & $\geq .95$ & $\geq .95$ & $\geq .95$ & $\geq .80$ & $\geq .70$ & el menor \\
\hline $\begin{array}{l}\text { Aceptable } \\
\text { Modelos }\end{array}$ & $\mathrm{p}>.06$ & $<.08$ & $\geq .90$ & $\geq .90$ & $\geq .90$ & $\geq .90$ & $\geq .90$ & $\geq .90$ & $\geq .70$ & $\geq .60$ & el menor \\
\hline Un factor & 5,185.03.000 & .084 & .857 & .827 & .780 & .757 & .773 & .905 & .706 & .700 & $5,317.03$ \\
\hline Cuatro factores & 6,233.52.000 & .093 & .850 & .819 & .734 & .707 & .727 & .909 & .667 & .661 & $6,363.52$ \\
\hline Cuatro factores bis & $3,410.67 .000$ & .068 & .912 & .893 & .858 & .843 & .851 & .900 & .773 & .766 & $3,544.67$ \\
\hline Bifactor & $3,000.54 .000$ & .063 & .921 & .904 & .877 & .864 & .869 & .905 & .793 & .786 & $3,132.54$ \\
\hline
\end{tabular}

Tabla 5

Consistencia interna, fiabilidad test-retest al cabo de un mes y correlaciones con el ASI-3 del ISRA-B

\begin{tabular}{|c|c|c|c|c|}
\hline $\begin{array}{l}\text { Consistencia Interna } \\
(\mathrm{n}=3,346)\end{array}$ & & & $\begin{array}{l}\text { Test-retest } \\
(\mathrm{n}=201)\end{array}$ & $\begin{array}{l}\text { ASI-3 } \\
(n=936)\end{array}$ \\
\hline & & Alfa & $\mathrm{r}$ & $r$ \\
\hline \multirow[t]{10}{*}{ Total ISRA-B } & & .94 & .78 & .61 \\
\hline & Total Respuestas & .91 & .73 & .58 \\
\hline & Cognitivo & .84 & .77 & .48 \\
\hline & Fisiológico & .86 & .61 & .54 \\
\hline & Motor & .70 & 69 & .49 \\
\hline & Total Situaciones & .89 & .73 & .56 \\
\hline & Evaluación & .82 & .69 & .46 \\
\hline & Interpersonal & .74 & .73 & .46 \\
\hline & Fóbicas & .69 & .75 & .43 \\
\hline & Cotidianas & .74 & .70 & .50 \\
\hline
\end{tabular}

transformadas en puntuaciones centiles y en cuartiles. Para calcular Cotidianas se han añadido s3 y s16 a la fórmula tradicional (s2, s5, s21, s22), de acuerdo con el AFC de este estudio.

\section{Discusión}

La ansiedad es la reacción emocional que más atención ha obtenido en la literatura científica, quizás porque es la que más se manifiesta en la vida cotidiana de los individuos, como una respuesta normal que nos prepara para afrontar la amenaza de un posible resultado negativo, y también la que más implicada está en la psicopatología de los trastornos emocionales (CanoVindel, 2011b, 2011a; González-Blanch et al., 2018a). Todo ello ha acarreado el desarrollo y análisis de un enorme arsenal de instrumentos de evaluación en el ámbito clínico (Cano-Vindel et al., 2018; González-Blanch et al., 2018b; Muñoz-Navarro et al., 2017a). Pero la ansiedad constituye también una reacción emocional normal en todas las esferas de la vida, por lo que se han desarrollado y estudiado un gran número de inventarios de propósito general (García-Batista et al., 2017) o específico (Furlan, SánchezRosas, 2018). Aunque la ansiedad ha sido mucho más estudiada como un constructo unidimensional, bien como rasgo general de 
Tabla 6

Correlaciones entre el ISRA-B y el ISRA original $(n=63$ )

\begin{tabular}{|c|c|c|c|c|c|c|}
\hline & & & \multirow[b]{2}{*}{ Cognitivo } & \multicolumn{3}{|c|}{ ISRA original (224) } \\
\hline & & & & Fisiológico & Motor & ISRA Total \\
\hline \multirow[t]{11}{*}{ Total ISRA-B (46) } & & & & & & .87 \\
\hline & Total Respuestas (24) & & & & & .84 \\
\hline & & Cognitivo & .77 & & & \\
\hline & & Fisiológico & & .82 & & \\
\hline & & Motor & & & .80 & \\
\hline & Total Situaciones (22) & & & & & .83 \\
\hline & & & Evaluación & Interpersonal & Fóbicas & Cotidianas \\
\hline & & Evaluación & .80 & & & \\
\hline & & Interpersonal & & .74 & & \\
\hline & & Fóbicas & & & .79 & \\
\hline & & Cotidianas & & & & .76 \\
\hline
\end{tabular}

Tabla 7

Capacidad de discriminación de la escala Total ISRA-B: población general vs. clínicos (pánico y agorafobia) por sexo

\begin{tabular}{|c|c|c|c|c|c|c|c|}
\hline \multirow[b]{2}{*}{ Variable } & \multirow[b]{2}{*}{ Población } & \multirow[b]{2}{*}{ Sexo } & \multirow[t]{2}{*}{ Media } & \multirow[t]{2}{*}{ Error Estándar } & \multicolumn{2}{|c|}{ Intervalo de confianza al 95\% } & \multirow[b]{2}{*}{$\mathrm{N}$} \\
\hline & & & & & Límite inferior & Límite superior & \\
\hline \multirow[t]{4}{*}{ Total ISRA-B } & General & Varones & 47.14 & .733 & 45.70 & 48.58 & 935 \\
\hline & & Mujeres & 61.90 & .679 & 60.57 & 63.23 & 1,090 \\
\hline & Clínicos & Varones & 85.85 & 2.895 & 80.17 & 91.53 & 60 \\
\hline & & Mujeres & 99.75 & 2.022 & 95.78 & 103.71 & 123 \\
\hline
\end{tabular}

\section{Tabla 8}

Capacidad de discriminación del ISRA-B entre los distintos grupos del estudio $(n=3,336)$ por sexo

\begin{tabular}{|c|c|c|c|c|c|}
\hline \multicolumn{6}{|c|}{ Total ISRA-B } \\
\hline \multirow[b]{2}{*}{ Grupo } & \multirow[b]{2}{*}{ Sexo } & \multirow[b]{2}{*}{ Media } & \multirow[b]{2}{*}{ Error estándar } & \multicolumn{2}{|c|}{ Intervalo de confianza al 95\% } \\
\hline & & & & Límite inferior & Límite superior \\
\hline \multirow[t]{2}{*}{ Población general } & Hombres & 47.14 & .747 & 45.68 & 48.60 \\
\hline & Mujeres & 61.90 & .692 & 60.54 & 63.26 \\
\hline \multirow[t]{2}{*}{ Psicosomáticos } & Hombres & 62.86 & 4.984 & 53.08 & 72.63 \\
\hline & Mujeres & 8.63 & 4.662 & 71.48 & 89.77 \\
\hline \multirow[t]{2}{*}{ Clínicos } & Hombres & 93.16 & 2.112 & 89.02 & 97.30 \\
\hline & Mujeres & 101.57 & 1.737 & 98.16 & 104.97 \\
\hline \multirow[t]{2}{*}{ Cáncer } & Hombres & & & & \\
\hline & Mujeres & 45.41 & 2.085 & 41.32 & 49.50 \\
\hline \multirow[t]{2}{*}{ Artritis Reumatoide } & Hombres & 57.50 & 7.223 & 43.34 & 71.66 \\
\hline & Mujeres & 77.33 & 4.170 & 69.16 & 85.51 \\
\hline \multirow[t]{2}{*}{ Parkinson } & Hombres & 88.24 & 4.984 & 78.47 & 98.01 \\
\hline & Mujeres & 96.41 & 4.870 & 86.86 & 105.96 \\
\hline \multirow[t]{2}{*}{ Estudiantes Psicología } & Hombres & 58.28 & 2.149 & 54.07 & 62.50 \\
\hline & Mujeres & 72.44 & .889 & 70.70 & 74.19 \\
\hline
\end{tabular}

ansiedad o como una reacción puntual o estado ansioso unidimensional, evaluado principalmente por el STAI; sin embargo, el estudio de las diferentes facetas, frente a la concepción unitaria, como reacción emocional en los tres sistemas de respuesta que propuso Lang (C, F y M) y ante diferentes situaciones o contextos, los rasgos específicos de ansiedad propuestos por Endler, ha tenido un buen desarrollo en los últimos 50 años (Martínez-Monteagudo et al., 2012), lo que ha llevado a la creación y estudio de diferentes instrumentos de evaluación que miden dichas facetas, útiles para diferentes propósitos (Chust-Hernández et al., 2019; GarcíaFernández et al., 2011). Entre estos se encuentra el ISRA, del que se ha derivado la versión abreviada ISRA-B, que analizamos en este trabajo y que evalúa las mismas 8 facetas diferentes de la ansiedad que la prueba original: $\mathrm{T}$ o nivel general de ansiedad (46 ítems), los tres sistemas de respuesta (C, F y M) y cuatro áreas situacionales o rasgos específicos de ansiedad (Evaluación o Test, Interpersonal o Social, Fóbicas y Cotidianas). Se puede usar sólo la escala $\mathrm{R}$ que proporciona una medida de rasgo general de ansiedad, así como los tres sistemas de R. También se puede usar sólo la escala $\mathrm{S}$ que mide el nivel general de ansiedad ante situaciones de alta frecuencia, lo que sería también similar a una medida de rasgo general, así como los cuatro rasgos específicos de ansiedad.

Los resultados de este estudio mostraron datos excelentes para la consistencia interna del ISRA-B en el total de la prueba (T) y la escala $\mathrm{R}$, que también se corroboraron en el estudio de México (González-Ramírez et al., 2014) y que alcanzaron valores similares calificados de buenos en Argentina (Medrano et al., 2017). La consistencia interna de la escala $\mathrm{S}$ es similar, algo menor, que la de $\mathrm{R}$ en este estudio y en los dos latinoamericanos, aunque está en la parte alta de la categoría "buena consistencia». Cuando se analizó el contenido de los ítems de las subescalas de R, en este estudio se obtuvo también buena consistencia para los sistemas Cy F, así como aceptable para $\mathrm{M}$. En el análisis de las subescalas de $\mathrm{S}$, también fue buena la consistencia de la subescala de ansiedad de Evaluación y aceptable para Interpersonal o social y Cotidianas, pero obtuvieron resultados muy ligeramente por debajo del punto crítico Fóbicas. Estos resultados para las subescalas de $\mathrm{R}$ y de $\mathrm{S}$ son similares a los encontrados en México (González-Ramírez et al., 2014) y Argentina (Medrano et al., 2017).

La validez convergente del ISRA-B ha obtenido excelentes resultados cuando se ha relacionado este instrumento con el original 
Tabla 9

Baremos de población general para mujeres

\begin{tabular}{|c|c|c|c|c|c|c|c|c|c|c|c|c|}
\hline & & Cognitivo & Fisiológico & Motor & Total R & Evaluación & Interpersonal & Fóbicas & Cotidianas & Total S & Total 46 & Cuartiles \\
\hline \multirow[t]{2}{*}{$\mathrm{N}$} & Válido & 1,090 & 1,090 & 1,090 & 1,090 & 1,090 & 1,090 & 1,090 & 1,090 & 1,090 & 1,090 & \\
\hline & Perdid. & 0 & 0 & 0 & 0 & 0 & 0 & 0 & 0 & 0 & 0 & \\
\hline Media & 11.34 & 8.28 & 7.81 & 27.44 & 14.79 & 4.64 & 6.86 & 8.17 & 34.46 & 61.90 & & \\
\hline Desviac. & 5.28 & 6.49 & 4.36 & 13.02 & 5.28 & 3.34 & 4.13 & 3.95 & 13.19 & 23.30 & & \\
\hline Mínimo & 0 & 0 & 0 & 0 & 1 & 0 & 0 & 0 & 2 & 4 & & \\
\hline Máximo & 27 & 32 & 22 & 73 & 28 & 16 & 20 & 19 & 74 & 127 & & \\
\hline \multirow[t]{20}{*}{ Percent. } & 99 & 24 & 27 & 20 & 63 & 26 & 13 & 18 & 19 & 67 & 120 & \\
\hline & 95 & 21 & 21 & 15 & 51 & 23 & 11 & 15 & 18 & 58 & 102 & \\
\hline & 90 & 18 & 18 & 14 & 44 & 22 & 9 & 12 & 15 & 52 & 93 & \\
\hline & 85 & 17 & 15 & 13 & 41 & 21 & 8 & 11 & 14 & 48 & 87 & \\
\hline & 80 & 16 & 14 & 11 & 38 & 20 & 8 & 10 & 12 & 45 & 82 & \\
\hline & 75 & 15 & 12 & 11 & 36 & 19 & 7 & 9 & 12 & 43 & 78 & Q3 \\
\hline & 70 & 14 & 11 & 10 & 34 & 18 & 6 & 9 & 11 & 42 & 75 & \\
\hline & 65 & 13 & 10 & 9 & 32 & 17 & 6 & 8 & 10 & 40 & 71 & \\
\hline & 60 & 13 & 9 & 9 & 30 & 17 & 5 & 7 & 10 & 38 & 67 & \\
\hline & 55 & 12 & 8 & 8 & 28 & 16 & 5 & 7 & 9 & 36 & 64 & \\
\hline & 50 & 11 & 7 & 8 & 26 & 15 & 4 & 7 & 8 & 34 & 61 & Q2 \\
\hline & 45 & 11 & 6 & 7 & 25 & 14 & 4 & 6 & 8 & 32 & 58 & \\
\hline & 40 & 10 & 5 & 6 & 23 & 13 & 3 & 5 & 7 & 31 & 55 & \\
\hline & 35 & 9 & 5 & 6 & 21 & 13 & 3 & 5 & 7 & 29 & 51 & \\
\hline & 30 & 8 & 4 & 5 & 19 & 12 & 3 & 4 & 6 & 28 & 48 & \\
\hline & 25 & 8 & 3 & 4 & 18 & 11 & 2 & 4 & 6 & 25 & 45 & Q1 \\
\hline & 20 & 7 & 2 & 4 & 15 & 10 & 2 & 3 & 5 & 23 & 42 & \\
\hline & 15 & 6 & 2 & 3 & 13 & 9 & 1 & 3 & 5 & 20 & 38 & \\
\hline & 10 & 4 & 1 & 2 & 11 & 8 & 0 & 2 & 4 & 17 & 31 & \\
\hline & 5 & 3 & 0 & 1 & 8 & 6 & 0 & 1 & 3 & 13 & 24 & \\
\hline
\end{tabular}

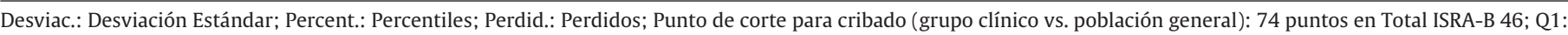
Cuartil 1; Q2: Cuartil 2; Q3, Cuartil 3.

Tabla 10

Baremos de población general para hombres

\begin{tabular}{|c|c|c|c|c|c|c|c|c|c|c|c|c|}
\hline & & Cognitivo & Fisiológico & Motor & Total R & Evaluación & Interpersonal & Fóbicas & Cotidianas & Total S & Total 46 & Cuartiles \\
\hline \multirow[t]{2}{*}{$\mathrm{N}$} & Válido & 935 & 935 & 935 & 935 & 935 & 935 & 935 & 935 & 935 & 935 & \\
\hline & Perdid. & 0 & 0 & 0 & 0 & 0 & 0 & 0 & 0 & 0 & 0 & \\
\hline Media & 8.95 & 5.52 & 5.94 & 2.41 & 11.90 & 3.97 & 4.23 & 6.63 & 26.73 & 47.14 & & \\
\hline Desviac. & 4.68 & 5.55 & 3.79 & 11.26 & 4.97 & 2.85 & 3.37 & 361 & 11.95 & 2.91 & & \\
\hline Mínimo & 0 & 0 & 0 & 0 & 1 & 0 & 0 & 0 & 2 & 4 & & \\
\hline Máximo & 24 & 26 & 19 & 59 & 26 & 14 & 17 & 21 & 74 & 102 & & \\
\hline \multirow[t]{20}{*}{ Percent. } & 99 & 21 & 24 & 15 & 52 & 24 & 12 & 14 & 16 & 55 & 95 & \\
\hline & 95 & 17 & 17 & 13 & 41 & 20 & 9 & 11 & 13 & 47 & 85 & \\
\hline & 90 & 15 & 14 & 11 & 37 & 18 & 8 & 9 & 11 & 43 & 76 & \\
\hline & 85 & 14 & 11 & 10 & 32 & 17 & 7 & 8 & 10 & 39 & 71 & \\
\hline & 80 & 13 & 10 & 9 & 30 & 16 & 6 & 7 & 10 & 37 & 65 & \\
\hline & 75 & 12 & 9 & 8 & 28 & 15 & 6 & 6 & 9 & 35 & 61 & Q3 \\
\hline & 70 & 11 & 8 & 8 & 26 & 14 & 5 & 6 & 8 & 33 & 57 & \\
\hline & 65 & 11 & 7 & 7 & 24 & 14 & 5 & 5 & 8 & 31 & 55 & \\
\hline & 60 & 10 & 5 & 7 & 22 & 13 & 4 & 5 & 8 & 30 & 52 & \\
\hline & 55 & 9 & 4 & 6 & 21 & 12 & 4 & 4 & 7 & 28 & 49 & \\
\hline & 50 & 9 & 4 & 6 & 19 & 12 & 4 & 4 & 6 & 26 & 46 & Q2 \\
\hline & 45 & 8 & 3 & 5 & 18 & 11 & 3 & 3 & 6 & 25 & 43 & \\
\hline & 40 & 8 & 2 & 4 & 16 & 11 & 3 & 3 & 5 & 23 & 41 & \\
\hline & 35 & 7 & 2 & 4 & 15 & 10 & 3 & 2 & 5 & 22 & 39 & \\
\hline & 30 & 6 & 2 & 4 & 13 & 9 & 2 & 2 & 4 & 20 & 35 & \\
\hline & 25 & 6 & 1 & 3 & 12 & 8 & 2 & 2 & 4 & 18 & 32 & Q1 \\
\hline & 20 & 5 & 1 & 2 & 10 & 8 & 1 & 1 & 3 & 16 & 28 & \\
\hline & 15 & 3 & 0 & 2 & 8 & 7 & 1 & 1 & 3 & 13 & 24 & \\
\hline & 10 & 4 & 0 & 1 & 6 & 5 & 0 & 0 & 2 & 11 & 19 & \\
\hline & 5 & 2 & 0 & 0 & 4 & 4 & 0 & 0 & 1 & 8 & 14 & \\
\hline
\end{tabular}

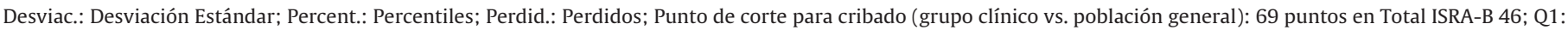
Cuartil 1; Q2: Cuartil 2; Q3, Cuartil 3.

ISRA, que a su vez mostró una alta correlación entre T y el STAI, en la población española. Además, en este estudio se comprobó que existía una fuerte asociación entre las medidas del ISRA-B y las del Î́ndice de Sensibilidad a la Ansiedad, versión 3, en la población española, como se encontró en México (González-Ramírez et al., 2014); también obtuvo una asociación positiva y significativa con el Estrés Percibido en la población argentina (Medrano et al., 2017), mostrando en todos los casos evidencias de validez externa.
La estructura factorial del ISRA-B obtenida, como suponíamos, no se ajustó a un modelo unidimensional de las reacciones emocionales o R de ansiedad, ni tampoco a un modelo de rasgo general de ansiedad, independiente de las $\mathrm{S}$, sino que se adecuó de forma aceptable al modelo de Lang (Lang, 1968; Martínez-Monteagudo et al., 2012), que defiende la existencia de los tres sistemas de R emocional, y al modelo de Endler de la interacción SxR, que defiende diferencias en el rasgo general asociadas a tipos de $S$ o rasgos específicos de ansiedad (Cano-Vindel, 1989; Endler, Okada, 1975), los dos 
modelos en los que se basa este instrumento. Además, se aceptaron modelos bifactor en ambos casos, lo que implica que se pueden usar las escalas globales, total $\mathrm{R}$ y total $\mathrm{S}$, aunque no añaden un gran porcentaje de varianza si ya se usan los factores específicos de $\mathrm{R}$ y de $\mathrm{S}$, con modelos de 3 y 4 factores respectivamente. Estos resultados en conjunto son similares a los obtenidos en México (González-Ramírez et al., 2014) y Argentina (Medrano et al., 2017).

El modelo de tres factores de $\mathrm{R}$ emocional ajustó de forma aceptable, pero no óptima, y en el AFE si bien todos los ítems obtuvieron pesos significativos en su factor, algunos presentaron ciertos problemas de covariabilidad. En el AFC los parámetros se ajustaron a los criterios mínimos comúnmente aceptados; sin embargo, en futuros estudios podría plantearse reducir aún más el ISRA-B, eliminando algunos ítems como, por ejemplo, r18, r7 y r6, que presentan mayor covariabilidad entre factores y pesos más bajos, para intentar conseguir un ajuste óptimo y con menos ítems.

A su vez, el modelo de cuatro factores de $\mathrm{S}$ o rasgos específicos de ansiedad tampoco ajustó de forma óptima, aunque sí aceptable, pero se hizo una reformulación del modelo para incluir las situaciones S3 y S16, que hasta ahora no se sumaban en las fórmulas de corrección de los rasgos específicos, entre las situaciones Cotidianas. En futuros estudios, también debería plantearse mejorar este ajuste y analizar la invarianza. También habría que revisar las fórmulas de las áreas situacionales en el ISRA original, utilizando AFC.

Aunque el ISRA-B no pretende ser una prueba de cribado, sino un instrumento de evaluación general de la ansiedad, que evalúa diversas facetas de la misma, su validez discriminante ha mostrado ser buena con las pruebas de curva COR o ROC. Para un propósito específico de cribado existen pruebas más cortas y con mayor validez discriminante, como el GAD-7 o incluso el GAD-2 (Cano-Vindel et al., 2018; Muñoz-Navarro et al., 2017a; Muñoz-Navarro et al., 2017b).

La capacidad de discriminación del ISRA-B ha sido demostrada al analizar las diferencias entre grupos y sexo. Se han obtenido medias en los niveles de ansiedad de los distintos grupos que marcan diferencias similares a las encontradas con grupos semejantes evaluados con el ISRA original, con un tamaño del efecto grande para el factor grupo y pequeño para el sexo (García-Batista et al., 2015; García Batista y Cano-Vindel, 2014; Martínez-Monteagudo et al., 2012; Miguel-Tobal y Cano-Vindel, 1986, 2002).

Asimismo, se han presentado los datos normativos, diferenciados por sexo y grupo. Los valores centrales de este estudio son ligeramente más altos que los encontrados en México (GonzálezRamírez et al., 2014) y más similares a los de Argentina (Medrano et al., 2017).

Entre las limitaciones de este estudio cabe mencionar que, a pesar de que el ISRA-B es un instrumento adecuado para evaluar la ansiedad, se trata de un autoinforme, con todas las limitaciones de este método de medida, pero también sus ventajas. Además, aunque se presentan datos de una amplia muestra, no se llevó a cabo una aleatorización de los participantes, por lo que no es representativa de la población española. Sin embargo, las muestras analizadas son grandes. Los dos AFC realizados no arrojaron un ajuste óptimo sobre la estructura de ambas escalas $\mathrm{R}$ y $\mathrm{S}$, aunque sí aceptable. Además, no se han realizado estudios de invarianza, que sería recomendable llevar a cabo, especialmente para sexo o género y población general versus población clínica. La búsqueda de una versión aún más breve, conservando la posibilidad de evaluar todas las facetas de la ansiedad que evalúa este instrumento, tratando de conseguir un ajuste óptimo de los dos modelos, R y S, es sin duda un reto para seguir investigando en esta línea, pero por ahora el ISRA-B simplifica enormemente la complejidad de la evaluación de todas esas facetas de la ansiedad, con buenos resultados.

\section{Conclusiones}

En definitiva, en el presente estudio se han presentado pruebas sobre las propiedades psicométricas del ISRA-B que indican que puede ser usado como una medida de la ansiedad confiable y válida de esta reacción emocional, tanto a nivel global (escala $\mathrm{R}$ ) como de los tres sistemas de respuesta (C, F y M). Por otro lado, también puede ser usado para evaluar de forma confiable y válida el nivel general de ansiedad ante $22 \mathrm{~S}$ diferentes (escala $\mathrm{S}$ ), algo que permite una medida alternativa al rasgo general de ansiedad (que se evalúa haciendo referencia en las instrucciones a «evalúe su nivel de ansiedad en cualquier situación»), además de permitir la evaluación de la ansiedad mediante cuatro rasgos específicos, en contextos de evaluación (test anxiety), en situaciones interpersonales o sociales, ante situaciones fóbicas y en situaciones de la vida cotidiana. Esta última escala, que era la más débil en número de ítems y propiedades psicométricas, se ha visto fortalecida por los resultados de este estudio. Por todo ello, consideramos que el ISRA-B es un instrumento válido y confiable, que mejora su aplicabilidad respecto al ISRA original, al simplificar formato y número de ítems, conservando sus propiedades psicométricas, por lo que se recomienda su uso.

\section{Financiación}

Esta investigación no recibió ninguna subvención específica de agencias de financiación en los sectores público, comercial o sin fines de lucro.

\section{Agradecimientos}

Esta investigación se ha llevado a cabo bajo el patrocinio económico parcial del proyecto de investigación "Evaluación de coste-efectividad del tratamiento psicológico transdiagnóstico grupal para los desórdenes emocionales en atención primaria" financiado por el Ministerio Ciencia, Innovación y Universidades MICINN (ref. PID2019-107243RB-C21).

\section{Conflicto de intereses}

Los autores declaran no tener ningún conflicto de intereses

\section{Referencias}

Amorim-Gaudêncio, C., Roustan Guillón, G. y Sirgo Rodríguez, A. (2004). Evaluation of anxiety in chronic dermatoses: Differences between sexes. Interamerican Journal of Psychology, 38(1), 105-114. http://dx.doi.org/10.30849/rip/ijp.v38i1.846

Aparicio-García, M. E., Fernández-Castilla, B., Giménez-Páez, M. A., Piris-Cava, E. y Fernández-Quijano, I. (2018). Influence of feminine gender norms in symptoms of anxiety in the Spanish context. Ansiedad y Estrés, 24(2-3), 60-66. http://dx.doi.org/10.1016/j.anyes.2018.03.001

Byrne, B. M. (2001). Structural equation modeling with AMOS EQS, and LISREL: Comparative approaches to testing for the factorial validity of a measuring instrument. International Journal of Testing, 1(1), 55-86. http://dx.doi.org/10.1207/s15327574ijt0101_4

Calvo, M. G., Avero, P., Castillo, M. D. y Miguel-Tobal, J. J. (2003). Multidimensiona anxiety and content-specificity effects in preferential processing of threat. European Psychologist, 8(4), 252-265. http://dx.doi.org/10.1027/1016-9040.8.4.252

Camuñas, N., Cano-Vindel, A., Pérez-Nieto, M. A. y González-Ordi, H. (2002). Inventario de Atribuciones Causales ante Exámines-IACE: Propiedades psicométricas. Ansiedad y Estrés, 8(2-3), 183-192.

Cano-Vindel, A. (1989). Cognición, emoción y personalidad: un estudio centrado en la ansiedad. Madrid: Universidad Complutense de Madrid.

Cano-Vindel, A. (2011a). Los desórdenes emocionales en Atención Primaria. Ansiedad y Estrés, 17(1), 75-97.

Cano-Vindel, A. (2011b). Bases teóricas y apoyo empírico de la intervención psicológica sobre los desórdenes emocionales en atención primaria. Una actualización. Ansiedad y Estrés, 17(2-3), 157-184.

Cano-Vindel, A. y Espada Largo, F. J. (2003). Fobia social y fobia simple: diferencias y similitudes en las manifestaciones clínicas. Ansiedad y Estrés, 9(1), 49-57. 
Cano-Vindel, A., Fernández,J. C. y Spielberger, C. D. (2012). La experiencia y expresión de la ansiedad y la ira en pacientes con asma bronquial. Ansiedad y Estrés, 18(2-3), 221-230.

Cano-Vindel, A. y Fernández Rodríguez, J. C. (1999). Perfiles de ira y ansiedad en el asma bronquial. Ansiedad y Estrés, 5(1), 25-35.

Cano-Vindel, A. y Macías, Y. (2002). Ansiedad, ira y tristeza-depresión en la enfermedad de Parkinson. Ansiedad y Estrés, 8(2-3), 245-255.

Cano-Vindel, A. y Miguel-Tobal, J.J. (1999). Evaluación de la ansiedad desde un enfoque interactivo y multidimensional: el Inventario de Situaciones y Respuestas de Ansiedad-ISRA. Psicología Contemporánea, 6, 14-21.

Cano-Vindel, A., Muñoz-Navarro, R., Medrano, L. A., Ruiz-Rodríguez, P., GonzálezBlanch, C., Gómez Castillo, M. D. y Santolaya, F. (2018). A computerized version of the Patient Health Questionnaire-4 as an ultra-brief screening tool to detect emotional disorders in primary care. Journal of Affective Disorders, 234, 247-255. http://dx.doi.org/10.1016/j.jad.2018.01.030

Chust-Hernández, P., Castellano-Rioja, E., Fernández-García, D. y Chust-Torrent, J. I. (2019). Ansiedad ante los exámenes en estudiantes de Enfermería: factores de riesgo emocionales y de sueño. Ansiedad y Estres, 25(2), 125-131. http://dx.doi.org/10.1016/j.anyes.2019.05.001

Endler, N. S. y Okada, M. (1975). A multidimensional measure of trait anxiety: The S-R inventory of general trait anxiousness. Journal of Consulting and Clinical Psychology, 43(3), 319-329. http://dx.doi.org/10.1037/h0076643

Entradas Saavedra, M., Ferreiro Cotorruelo, F. J., Folk Madrid, A. y Marín Fuste, J. (1992). Social skills: An experience with psychiatric patients in out-patient care [Habilidades sociales: una experiencia con pacientes psiquiátricos en consultas ambulatorias]. Anales de Psiquiatría, 8(7), 282-285.

Fernández Rodríguez, J. C. F. (2012). The experience and expression of anger and anxiety in bronchial asthma patients. Anuario de Psicología, 42(2), 213-225.

Furlan, L. y Sánchez-Rosas, J. (2018). Evidencias de validez y confiabilidad de una Escala de Evitación Conductual en Exámenes Orales en estudiantes universitarios. Ansiedad y Estrés, 24(2-3), 90-98. http://dx.doi.org/10.1016/J.ANYES.; $1 ; 2018.05 .001$

García-Batista, Z. E., Cano-Vindel, A. y Herrera-Martínez, S. X. (2015). Propiedades psicométricas del Inventario de Situaciones y Respuestas de Ansiedad (ISRA) en una muestra dominicana. Pensamiento Americano, 8(15), 56-66. http://dx.doi.org/10.21803/penamer.8.15.326

García-Batista, Z. E., Guerra-Peña, K., Cano-Vindel, A., Herrera-Martínez, S. X., Flores-Kanter, P. E. y Medrano, L. A. (2017). Propiedades psicométricas del Inventario de ansiedad estado-rasgo en población general y hospitalaria de República Dominicana. Ansiedad y Estrés, 23(2-3), 53-58. http://dx.doi.org/10.1016/j.anyes.2017.09.004

García-Fernández, J. M., Inglés, C. J., Martínez-Monteagudo, M. C., Marzo, J. C. y Estévez, E. (2011). Inventario de Ansiedad Escolar: validación en una muestra de estudiantes de Educación Secundaria. Psicothema, 23(2), 301-307.

García-Naveira Vaamonde, A. y Ruiz-Barquín, R. (2015). Adaptation of ISRA-B to performance football players [Adaptación del ISRA-B a la población deportiva a través de jugadores de fútbol de rendimiento]. Ansiedad y Estrés, 21(1), 83-94.

García-Torres, F., Alós, F. J. y Castillo-Mayén, R. (2018). Identification of the anxiety response pattern in short-term breast cancer survivors [Identificación del patrón de respuesta a la ansiedad en las supervivientes a corto plazo del cáncer de mama]. Anuario de Psicología, 48(2), 59-63. http://dx.doi.org/10.1016/j.anpsic.2018.07.003

García Batista, Z. y Cano-Vindel, A. (2014). Estandarización y validación del Inventario de Situaciones y Respuestas de Ansiedad (ISRA) en la población de República Dominicana. Summa Psicológica UST, 11(2), 81-99, http://www.summapsicologica.cl/index.php/summa/article/view/130.

González-Blanch, C., Umaran-Alfageme, O., Cordero-Andrés, P., Muñoz-Navarro, R., Ruiz-Rodríguez, P., Medrano, L. A. y Cano-Vindel, A. (2018). Tratamiento psicológico de los trastornos emocionales en Atención Primaria: el manual de tratamiento transdiagnóstico del estudio PsicAP. Ansiedad y Estrés, 24(1), 1-11. http://dx.doi.org/10.1016/j.anyes.2017.10.005

González-Blanch, C., Medrano, L. A., Muñoz-Navarro, R., Ruíz-Rodríguez, P., Moriana, J. A., Limonero, J. T. y Cano-Vindel, A. (2018). Factor structure and measurement invariance across various demographic groups and over time for the PHQ-9 in primary care patients in Spain. PLoS One, 13(2), e0193356. http://dx.doi.org/10.1371/journal.pone.0193356

González-Ramírez, M. T. Quezada-Berumen, L., del, C., Díaz-Rodríguez, C. L. y CanoVindel, A. (2014). Adaptación para México y estructura factorial del inventario de situaciones y respuestas de Ansiedad breve (ISRA-B). Ansiedad y Estrés, 20(1), $89-100$.

González Ordi, H. (1994). Sugestión, sugestionabilidad y ansiedad: Investigación e implicaciones clínicas. Ansiedad y Estrés, 0, 135-145.

Hu, L.-T. y Bentler, P. M. (1999). Cutoff criteria for fit indexes in covariance structure analysis: Conventional criteria versus new alternatives. Structural Equation Modeling: A Multidisciplinary Journal, 6(1), 1-55. http://dx.doi.org/10.1080/10705519909540118

Jurado Solorzano, A. M. (2010). Adaptación del Inventario de Situaciones y Respuestas de Ansiedad (ISRA) a la población de Costa Rica. Facultad de Psicología. Universidad Complutense de Madrid.

Lang, P. (1968). Fear reduction and fear behavior: problems in treating a construct. En J. M. Shlien (Ed.), Research in Psychotherapy. Chicago, Ill.: American Psychological Association.

Leibovich de Figueroa, N. B. (2001). ISRA. Inventario de Situaciones y Respuestas de Ansiedad. Buenos Aires, Arg.: TEA Ediciones.

Losiak, W. (2005). Shapes of anxiety: Analysis of anxiety profiles measured with S-R questionnaire. Ansiedad y Estrés, 11(2-3), 157-162.
Macías-Delgado, Y., Pedraza-Núñez, H., Jiménez-Morales, R., Pérez-Rodríguez, M., Valle-Solano, R.-C. y Fundara-Díaz, R. (2014). Sobrecarga en los cuidadores primarios de pacientes con esclerosis múltiple: su relación con la depresión y ansiedad. Revista Mexicana de Neurociencia, 15(2), 81-86.

Martínez-Monteagudo, M.-C., Inglés Saura, C. J., Cano-Vindel, A. y García-Fernández, J. M. (2012). Estado actual de la investigación sobre la teoría tridimensional de la ansiedad de Lang. Ansiedad y Estrés, 18(2-3), 201-219.

Martínez Sánchez, F., Cano-Vindel, A., Castillo Precioso, J. C., Sánchez García, J. A., Ortiz Soria, B. y Gordillo del Valle, E. (1995). Una Escala Reducida de Ansiedad basada en el Inventario de Situaciones y Respuestas de Ansiedad (ISRA): un estudio exploratorio. Anales de Psicología, 11(1), 97-104.

Medrano, L. A., Moretti, L. S., Muñoz-Navarro, R. y Cano-Vindel, A. (2017). Validación argentina de la versión breve del Inventario de Situaciones y Respuestas de Ansiedad. Psiencia. Revista Latinoamericana de Ciencia Psicológica, 9(4) http://dx.doi.org/10.5872/psiencia/9.4.41

Medrano, L. A., Muñoz-Navarro, R. y Cano-Vindel, A. (2016). Procesos cognitivos y regulación emocional: Aportes desde una aproximación psicoevolucionista. Ansiedad y Estrés, 22(2), 47-54. http://dx.doi.org/10.1016/j.anyes.2016.11.001

Miguel-Tobal, J. J. y Cano-Vindel, A. (1986). Inventario de situaciones y respuestas de ansiedad (ISRA): Manual. Buenos Aires, Argentina: TEA.

Miguel-Tobal, J. J. y Cano-Vindel, A. (1995). Differential profiles in anxiety disorders [Perfiles diferenciales de los trastornos de ansiedad]. Ansiedad y Estrés, 1(1), $37-60$.

Miguel-Tobal, J. J. y Cano-Vindel, A. (2002). Inventario de Situaciones y Respuestas de Ansiedad (ISRA). Buenos Aires, Argentina: TEA.

Miguel Tobal, F., Martín Díaz, M. D. y Navlet Salvatierra, M. R. (2001). Niveles de ansiedad en distintas modalidades deportivas. Ansiedad y Estrés, 7(1), 57-68.

Moreno-Rosset, C. y Martín Díaz, M. D. (2009). La ansiedad en parejas fértiles e infértiles. Ansiedad y Estrés, 15(1), 97-109.

Moretti, F., Galeazzi, A., Miguel-Tobal, J. J. y Cano-Vindel, A. (2006). Aplicación del ISRA-J en adolescentes. Revista Lusófona de Ciencias da Mente e do Comportamento, 8(1), 251-259.

Muñiz Fernández, J. y Fernández-Hermida, J. R. (2010). The opinion of Spanish psychologists on the use of tests [La opinión de los psicólogos españoles sobre el uso de los tests]. Papeles del Psicólogo, 31(1), 108-121, http://www.papelesdelpsicologo.es/pdf/1801.pdf.

Muñoz-Navarro, R., Cano-Vindel, A., Moriana, J. A., Medrano, L. A., Ruiz-Rodríguez, P., Agüero-Gento, L. y Ramírez-Manent, J. I. (2017). Screening for generalized anxiety disorder in Spanish primary care centers with the GAD-7. Psychiatry Research, 256, 312-317. http://dx.doi.org/10.1016/j.psychres.2017.06.023

Muñoz-Navarro, R., Cano-Vindel, A., Ruiz-Rodríguez, P., Medrano, L. A., GonzálezBlanch, C., Moriana, J. A. y Dongil-Collado, E. (2017). Modelo jerárquico de diagnóstico y derivación de los trastornos mentales comunes en centros de atención primaria. Una propuesta a partir del ensayo clínico PsicAP. Ansiedad y Estrés, 23(2-3), 124-129. http://dx.doi.org/10.1016/j.anyes.2017.10.002

Nieto Fernández, E. y Barraca Mairal, J. (2017). Behavioral activation versus cognitive restructuring to reduce automatic negative thoughts in anxiety generating situations. Psicothema, 29(2), 172-177.

Nogués Moyano, V., González Barrón, R. y Sifre García, S. (2000). Ansiedad y enfermedad inflamatoria intestinal. Ansiedad y Estrés, 6(2-3), 281-294.

Nunnally, J. C. y Bernstein, I. H. (1994). Psychometric theory. New York: McGraw-Hill.

Pérez-Nieto, M. A., Cano-Vindel, A., Sayalero, M. T., Camuñas, N., Miguel Tobal, J. J. y Blanco, J. M. (2001). La ansiedad, la ira y el estrés asistencial en el ámbito hospitalario: Un estudio sobre sus relaciones y la eficacia del tratamiento. Ansiedad y Estrés, 7, 247-257.

Pérez Pareja, F. J., Borrás Sansaloni, C. y Palmer Pol, A. L. (1994). Anxiety and stress as predictors of the premenstrual syndrome [Ansiedad y estrés como predictores del síndrome premenstrual]. Ansiedad y Estrés, 0, 65-76, 0.

Redondo Delgado, M. M., Miguel Tobal, J. J. y Pérez Nieto, M. A. (2007). El papel del tiempo de evolución de la artritis reumatoide en las emociones negativas. Ansiedad y Estrés, 13(2-3), 141-151.

Richaud de Minzi, M. C. y Sacchi, C. (1995). Estudio de un inventario de situaciones y respuestas de ansiedad con adultos jóvenes argentinos. Revista Interamericana de Psicología, 29(1), 65-74, http://0search.proquest.com.cisne.sim.ucm.es/docview $/ 618969884$ ? accountid $=14514$

Rodríguez López, J. A., González Ordi, H., Cano Vindel, A. y Iruarrizaga Díez, I. (2007). Ansiedad en drogodependientes: Diferencias existentes en función del programa de tratamiento. Ansiedad y Estrés, 13, 241-252.

Rodríguez Martín, B. C., Molerio Pérez, O., Martínez Rodríguez, L., González Paneca, C. L. y Navarro Otero, S. M. (2014). Estructura factorial, confiabilidad y validez del Inventario de Supresión del Oso Blanco en adultos cubanos. Alternativas Cubanas en Psicología, 2(6), 92-101.

Sánchez-López, M. P. y Dresch, V. (2008). The 12-Item General Health Questionnaire (GHQ-12): Reliability, external validity and factor structure in the Spanish population. Psicothema, 20(4), 839-843.

Sandín, B., Valiente, R. M., Chorot, P. y Santed Germán, M. A. (2007). ASI-3: Nueva escala para la evaluación de la sensibilidad a la ansiedad. Revista de Psicopatología y Psicología Clínica, 12(2), 91-104. http://dx.doi.org/10.5944/rppc.vol.12.num.2.2007.4036

Sanz Fernández, J. (1991). The specific traits of anxiety in the Anxiety Situations and Responses Inventory (ASRI): Construct validity and relationship to depression. Evaluación Psicológica, 7(2), 149-173, http://www.ucm.es/BUCM/checkip.php?/docview/618113772? accountid = 14514

Sanz, J. (2014). Recomendaciones para la utilización de la adaptación española del Inventario de Ansiedad de Beck (BAI) en la práctica clínica. Clínica y Salud, 25(1), 39-48. http://dx.doi.org/10.5093/cl2014a3 
Solano Pinto, N. y Cano-Vindel, A. (2012). Anxiety in eating disorders: A comparative study [Ansiedad en los trastornos alimentarios: un estudio comparativo]. Psicothema, 24(3), 384-389.

Spielberger, C. D., Gorsuch, R. L. y Lushene, E. (2010). STAI. Cuestionario de Ansiedad Estado-Rasgo. Argentina: TEA Ediciones.

Taylor, J. A. (1953). A personality scale of manifest anxiety. The Journal of Abnormal and Social Psychology, 48(2), 285-290.

Terol Cantero, M. A., López-Roig, S., Rodríguez-Marín, J., Aragón Gelabert, M. M., Pastor, M. A. y Reig, M. T. (2007). Propiedades psicométricas de la Escala Hospitalaria de Ansiedad y Depresión (HAD) en población Española. Ansiedad y Estrés, 13(2), 163-176.

Villada, C., Hidalgo, V., Almela, M. y Salvador, A. (2016). Individual differences in the psychobiological response to psychosocial stress (Trier Social Stress Test):
The relevance of trait anxiety and coping styles. Stress and Health, 32(2), 90-99. http://dx.doi.org/10.1002/smi.2582

Villada, C., Hidalgo, V., Almela, M. y Salvador, A. (2018). Assessing performance on an evaluated speaking task: the role of self-efficacy, anxiety, and cardiac autonomic reactivity. Journal of Psychophysiology, 32(2), 64-74. http://dx.doi.org/10.1027/0269-8803/a000185

Yorio, A., Pompilio, L., Marro, C., Leibovich de, F. N. y Segura, E. T. (1998). EEG asymmetries, heart rate, and personality traits relations [Asimetrías electroencefalográficas asociadas a variaciones cardiovasculares, rasgos de ansiedad y factores de personalidad]. Ansiedad y Estrés, 4(1), 43-50. 